\title{
La singular historia del Doctor Gusano -Nicholas Andry de Boisregard- y de sus hijas Parasitología y Ortopedia
}

\author{
Walter Ledermann
}

Hospital LuisCalvo Mackenna.

Recibido: 24 agosto de 2012

Correspondencia a: Walter Ledermann Dehnhardt oncemayor@gmail.com

\section{The singular story of Doctor Worm -Nicholas Andry de Boisregard- and of his daughters Parasitology and Orthopaedics}

Homini verminoso or Dr. Worm were the nicknames that Nicholas Andry won in life for his consecration to the study of intestinal worms and for his bad temper, which led him to fiercely attack the surgeons. The article reassumes the studies and contributions that gave Andry the title of Father of Parasitology and the candidacy to Father of Orthopaedics, and mentions some other candidates to this honor. Quite a man, he had -besides his biological one- two famous daughters, growing till our days; wrote at least three valuable books; and planted the immortal "tree of Andry", the symbol of Orthopaedics.

Key words: Nicholas Andry, Dr. Worm, Parasitology, Orthopaedics.

Palabras clave: Nicholas Andry, Dr. Gusano, Parasitología, Ortopedia.

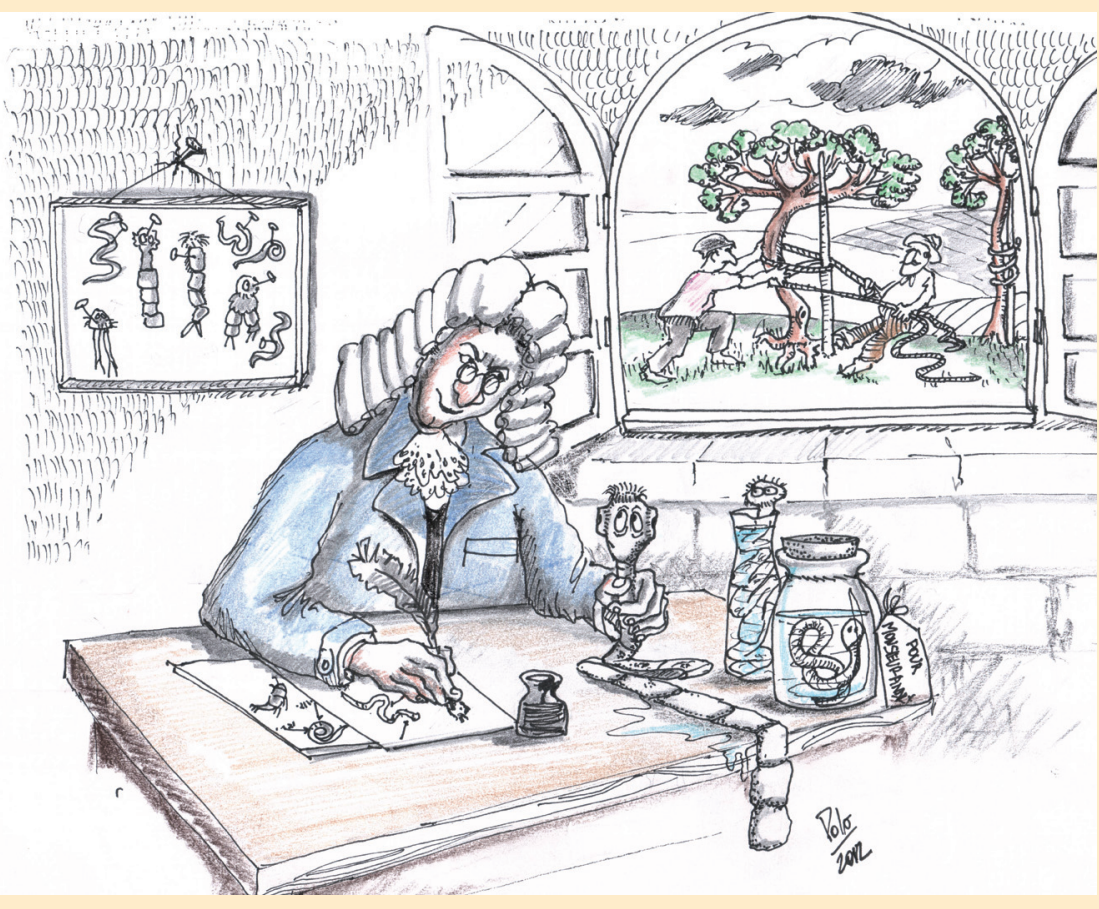

Introducción

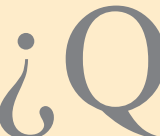

ué acciones, qué caminos, qué circunstancias pueden llevar a un médico a sufrir el mote de Doctor Gusano? "Gusano", en el lenguaje corriente y en el literario también, tiene una connotación de vileza y ruindad, derivada sin duda de la forma, desnuda y casi indiferenciada, de los vermes, así como de su movimiento arrastrado, de su vida ajena a la luz, subterránea y ciega, forma y método de traslación que recuerdan a las serpientes venenosas, todo lo cual nos produce instintivamente, si no temor, suficiente asco y rechazo. Se trata de gusano al hombre arrastrado y servil como al ínfimo y al despreciable; su ubicación en la naturaleza, para el hombre común, está en la sima del mundo animal, así como en el otro extremo, en la altura está el águila; sólo la araña puede producir mayor rechazo.

El médico tan desdeñosamente motejado no compartió esta acrimonia contra los vermes; no sintió, en sus palabras, vergüenza por deleitarse en su contemplación y estudio; su motivación nació, seguramente, al enfrentar en el ejercicio de su profesión la plaga que en aquella época representaban las infestaciones masivas por áscaris y oxiuros en la población de Europa. Creemos que, siendo un médico general, tuvo especial interés por sus pacientes infantiles: de no haber sido así... ¿cómo se explicaría, entonces, que se haya preocupado también de la ortopedia, por aquellos años inexistente y sin nombre? $\mathrm{Y}$, en tal creencia, como infectólogos y como pediatras, estimamos necesario divulgar su existencia y su obra entre nuestros colegas.

Nicholas Andry, así, a secas, que el título de Boisregard se lo agregó más tarde para parecer noble, nació en Lyon en 1658 , en el seno de una familia de regulares recursos. Su padre era un mercader sin mayor instrucción, pero se preocupaba de la educación de sus hijos y así pudo Nicholas a los 27 años ingresar a un seminario, movido por una vocación religiosa que luego se mostró harto débil, y por el ejemplo de sus dos hermanos teólogos, pero al poco tiempo lo tenemos como profesor en un colegio de 
Lyon, donde enseñaba humanidades. El hombre, que ya se hacía llamar Boisregard, tardaba en encontrar su vocación y poco duró como maestro, terminando por estudiar medicina en la Universidad de Reims, titulándose en 1697 , ya de 39 años, en otra universidad, en París. La teología y el humanismo, sin embargo, seguían presentes en su pensamiento y le dieron a su medicina un toque "humano", que ya se advierte en el título de su tesis de grado: An in morborum cura, hilaritas in medico, obedientia in aegro, que expone cómo en la curación de las enfermedades se relacionan -o deben hacerlo- la felicidad del médico con la obediencia de los pacientes ${ }^{1}$.

Hombre de variados intereses, se centró principalmente en dos, de donde nacieron sus dos "paternidades". Vayamos a ellas.

\section{Padre de la Parasitología}

Sobre su interés por los gusanos se han emitido varias hipótesis, siendo la más lógica y sencilla suponer que nació al observar la gran prevalencia de las infecciones parasitarias en su época. Por su parte, el conde húngaro Jules Andrassy, en un pie de página y con letra chica, transcribe el párrafo de una supuesta carta de Andry a su tocayo Hartsoeker, que centra este interés en una imagen en la infancia: Íbamos a menudo con mi padre a visitar a los huérfanos que cuidaban los dominicos en un hogar vecino a la iglesia, y como, impresionado por lo pálidos y delgados que se veían, así como por sus vientres abultados, preguntara una vez qué les pasaba, el Superior me dijo que estaban de esta manera "pues tenían los estómagos tan llenos de gusanos que les salían por las narices".

Andry no creía, como la mayoría de sus colegas, que los gusanos se generaban al interior de los enfermos, sosteniendo que estos parásitos eran engullidos con los alimentos. Pensaba que muchas enfermedades eran causadas y trasmitidas por ellos y, si no siempre se veían, era porque algunos tenían un tamaño muy pequeño para ser vistos a ojo desnudo, expresando: Si consideramos los Huevos de las Cuncunas, Moscas y otros pequeños insectos con el casi infinito número de esos pequeños Animales, que los Microscopios nos descubren en Licores (así dice claramente, "licores" y no "líquidos" ni "humores"), $y$ generalmente en todos los Cuerpos, encontraremos fácilmente que no hay nada en la Naturaleza dentro del cual la Semilla de Insectos no pueda introducirse, y que una gran Cantidad de ellos pueden entrar en el Cuerpo de un Hombre, al igual que en los de otros Animales, por medio de Aire o Alimentos. Entonces, ya que el calor es suficiente para sacar adelante los Gusanos contenidos en estos Huevos, cuando estos Huevos encuentran una Materia conveniente, es fácil entender que varias Especies de ellos pueden producirse en el Cuerpo de un Hombre de acuerdo a las diferentes Materias que encuentren alli... ${ }^{3}$.
Seamos benévolos y supongamos que el texto quiso hacer referencia a gusanos microscópicos, pero no nos queda muy claro. En todo caso, es decidor que Andry publicara entre otras esta carta del ya mencionado Nicolás Hartsoeker (1656-1725), investigador que postulara a algunos pequeños gusanos como causantes de enfermedades venéreas, lo que nos inclina a creer que el ortopedista y parasitólogo participaba de la creencia en los animalcula o semillas. La carta dice: Para decirle mis pensamientos, Sir, creo que Gusanos ocasionan la mayoría de las Enfermedades con que la Humanidad es atacada; y de este modo quienes tienen los Distempers que son llamados Venéreos, alimentan en sus Cuerpos un número infinito de Insectos invisibles, quienes crecen y devoran todo lo que encuentran en su Camino, y ocasionan todos los Desórdenes... ${ }^{4}$.

Pero al señor de Boisregard le interesaban más que los gusanos microscópicos, curiosos e hipotéticos causantes de algunas enfermedades infecciosas, los vermes macroscópicos, a cuyo estudio dedicó sus mejores años y cuyos resultados publicó en sendos libros, uno de ellos disponible in extenso en la red, cuyo larguísimo título nos hemos sentido tentados a presentarles en nuestra imperfecta traducción: Vermes solitarios y otros de diversas especies, de los cuales se trata en el libro de la generación de estos vermes, representados en varias láminas, con los reenvios a las páginas donde se ha hablado de ellos, donde se informan varias notas importantes sobre este tema. Más abajo hay una cita de Aristóteles, primero en griego y luego en su versión francesa: No se debe, por una vanidad pueril, tener vergüenza de contemplar la naturaleza en los más viles animales; ella no produce nada que no encierre motivos de admiración. Y luego, la detallada indicación del lugar donde fue impreso el libro, para que no se extravíe quien quiera comprarlo: En Paris, en los bajos de la calle Harpe, donde Laurent d'Houry, Impresor-Librero, al Saint Esprit, delante de la calle Saint Severin. M.DCC.XVIII. Con privilegio del Rey.

Pero, luego de leer atentamente este bello libro, cuya lectura recomendamos calurosamente a quienes se aburran en una tarde lluviosa de domingo, no encontramos nada sobre los minúsculos o finos gusanillos responsables del contagio y de la diseminación de las epidemias, visibles sólo con ayuda del microscopio, ellos se encuentran en el otro libro -el primero sobre el tema- que ¡hélas! no está indexado en Internet: De la generation des vers dans le corps de l'homme, de la nature et des espéces de cette maladie, de moyens de l'en préserver et de le guérir. Título que podríamos traducir como "De la generación de los gusanos en el cuerpo humano, la naturaleza y especies de esta enfermedad; los medios para evitarlos y curarlos ${ }^{3}$. En él se mencionan las cartas de Hartsoeker y también otra de Georges Bagliv: De la Generación de vermes en el cuerpo del hombre por Me. Nicolas Andry. 
Este Georges Blagiv o Blagivi (1668-1707), era un distinguido médico de la entonces República de Dubrovnik, pero que siendo adoptado por un caballero italiano de ese apellido, trabajó mayormente en Italia, dedicado al estudio de las fibras del cuerpo humano y a la circulación de sus humores, investigaciones que lo acercan a la escuela "mecanicista" de Giovanni Borelli. Discípulo de Malpighi, a quien practicó la autopsia (después de muerto, claro), era anatomista y microscopista experto; dejó textos hoy carentes de interés y fue miembro de la Royal Society ${ }^{6}$.

Examinando las láminas de la obra capital de Andry, donde junto a sus propios hallazgos aparecen dibujados gusanos que le enviaban colaboradores desde todo el mundo, creemos que no pocos intentaban tomarle el pelo con aterradores vermes con forma de homúnculos, fabricados quizás con qué material farináceo o gomoso, que el Dr. Gusano incluía en su obra sin más ni más. Falta sistematización y rigurosidad en la clasificación, y las tenias, áscaris y oxiuros aparecen sin orden, entremezclados con estos seres fabulosos enviados por los burlones. Aun así, y considerando el estado de las ciencias médicas en la época que le tocó vivir, Andry tiene méritos para elevar al menos su candidatura a Padre de la Parasitología.

\section{El Doctor Gusano}

Este interés fervoroso por los vermes y su afán de suponerlos causa de muchas enfermedades, aunque no se pudiera verlos, provocó irónicas reacciones entre sus colegas. Contribuyó a ellas su reconocido mal carácter, tanto que ha merecido los epítetos de soberbio, desdeñoso y rencoroso. Era hombre de ira fácil, como lo muestra una caricatura de la época, donde bajo el título de Homini verminoso aparece pateando la puerta de un cirujano-barbero para hacerle beber un purgante vermicida. Y, cuando Andry llevaba más de un siglo en su sepultura, la burla continúa y el médico naval Maurice Véritail, aludiendo a la carta de Hartsoecker que reprodujera en su obra, donde se habla de los "huevos de las cuncunas", suponiendo que estas larvas eran definitivamente gusanos, dice: Cazando infatigablemente a los gusanos, este sabio casi exterminó las mariposas de Francia...?

Nuestro profesor Eduardo Dussert, a quien debemos el conocimiento de este libro, que alguna vez estuvo en nuestras manos y cuyo paradero actual desconocemos, lo tenía en mucha estima y lo consideraba extremadamente gracioso, si bien nos confesara que el Dr. Véritail tenía poco de veritas y le parecía más un aventurero fantasioso y embustero que un médico verdadero, sosteniendo que habría servido de modelo para un personaje de la picaresca española. Tras larga búsqueda en la literatura española de los siglos XVIII y XIX, terminamos por encontrar en el siglo veinte al escritor León Villanúa y Artero con su héroe Ernest Vind Bernacle, médico de la Real Marina de
Inglaterra, quien aparece como estafador internacional en una de sus novelas más famosas ${ }^{8}$. Sin embargo, parece que el escritor confundió a Véritail con el "doctor Gusano", creyendo que el mote se debía a una naturaleza vil, y puso en boca del fabuloso Vind palabras más propias de Andry que de su difamador.

Al decir esto, aludimos a la desconfianza que Andry sentía hacia la medicina de su época y a su enemistad con los cirujano-barberos, reflejadas en las siguientes expresiones de Vind y actualizadas a la medicina de 1930:

- Es una ciencia desconocida hasta hoy día, se saben pocas cosas y lo demás es empirismo puro; la cirugía es algo mecánico, buena para los ingenieros del cuerpo humano.

¿Aludía a Borelli, a quien todavía no llegamos, y a la escuela iatro-matemática? ¿A Andry y la ortopedia? Y, más adelante, Villanúa pone en boca de un personaje que se hacía pasar por Vind, palabras odiosas y envenenadas: Los médicos que ejercen la profesión; los cirujanos que sierran, cortan y despedazan; siempre por dinero, claro es, son unos entes despreciables. La gloria será, al (correr del) tiempo, para los investigadores que recorren los hospitales en silencio anotando cosas, los que pasan las noches ante el microscopio mirando microorganismos, cuya vida no comprendéis vosotros, los clínicos, en vuestra tosquedad de recetas...

¿Qué tal? ¡Si pareciera que Andry hablara por su boca!

\section{Espermios, gusanos y Voltaire}

Se acusó en su momento a Hartsoeker, el corresponsal de Andry, de afirmar que había visto homúnculos dentro de los espermios, suponiendo que éstos eran pre-hombres, pero no hubo tal. También esta acusación salpicó a Andry, que en su obra Sobre la generación de vermes en el cuerpo humano los consideraba gusanos de una especial clase, explicando que si le extraía al perro un testículo y haciéndole luego varios cortes, podian visualizarse como veloces gusanillos avanzando por los vasos deferentes. Romi Kohler relata cómo Voltaire se burló de estos espermios de Andry en su novela L'homme aux quarante écus, describiéndolos como esos hombres pequeñitos que son tan buenos nadadores, agregando que el investigador pasa tanto tiempo buscando al hombre bajo su microscopio que lo reduce a una oruga ${ }^{9}$.

La obra escrita de Voltaire comprende 50 volúmenes de unas 600 páginas cada uno en la edición que hiciera Morland ${ }^{10} \mathrm{y}$ eso sin contar los dos gruesos tomos que conforman el índice. De los 50 volúmenes, 18 están dedicados solamente a las más de 10.000 cartas que escribiera este prodigioso filósofo: debemos agradecer a Kohler por el trabajo de encontrar entre tantísimo escrito la novela mencionada, que el Abate Marchena desechara incluir en el librito de Novelas escogidas de Voltaire que adorna nuestra biblioteca y cuya lectura recomendamos ${ }^{11}$. 


\section{Padre de la Ortopedia}

Para nuestra historia tenemos que remontarnos a Giovanni Augusto Borelli (1608-1679), que alguna vez postulara el contagio a través de minúsculos seres vivos, idea que por error suele adjudicarse a Pierre Borelli (16201689). Dice una enciclopedia ${ }^{12}$ que G.A. Borelli fue uno de los líderes de la secta iatro-matemática, o de quienes habían intentado aplicar las matemáticas a la medicina; y que en un tratado titulado De le cagione delle febri maligni di Sicilia, publicado en Cosenza en1649, hizo una relación de esta peste que la había asolado en 1647-1648, lo cual nos inclina a pensar que él y no Pierre fue quien postulara esta modalidad de contagio. Designado profesor de la Universidad de Pisa en 1656, fue pionero en intentar la aplicación de principios matemáticos para explicar el funcionamiento de órganos humanos, practicando para ello numerosas disecciones y publicando sus resultados en De motu animalium, en el cual descansa su fama médica y donde destaca la participación de la musculatura y de los huesos en la bipedoestación y en la marcha. Para nuestra especialidad, sin embargo, importa más su segundo tratado sobre las enfermedades infecciosas, publicado en 1658: Della causa delle febri maligni...

Sin duda Andry conocía la obra de Borelli y en ella encontró inspiración, relacionando la salud con el ejercicio, que fortalecía músculos y huesos. Esta preocupación por desarrollo muscular y la correcta posición del cuerpo, la tuvo nuestro héroe durante toda su vida, pero como el libro que creaba el término ortopedia y mostraba el árbol torcido que sería su emblema fue publicado a los 83 años de edad, apenas uno antes de su muerte, suele decirse que, aparte de escribir éste, nada hizo por esta disciplina, siendo más bien un anti-cirujano, olvidando muchas ingeniosas técnicas propuestas para tratar la deformidades óseas, que incluían varios tipos de corset hechos con barbas de ballena para corregir la escoliosis, con protuberancias sagazmente dispuestas para enderezar cada curvatura.

Estos ingeniosos y crueles corset, así como el mismo “árbol de Andry" (ya lo veremos, ya lo veremos), parecen benévolos juguetes al lado de los instrumentos de tortura usados para corregir la escoliosis en los siguientes siglos. E. Kirmissen, en un tratado de medicina infantil de comienzos del siglo XX muestra dibujos de varias máquinas de tracción y enderezamiento que ponen los pelos de punta, así como de suspensiones combinadas con presiones laterales; inclinaciones sobre un rodillo transversal y tracción; una tabla en plano inclinado, donde la víctima se acuesta y una pesa lo tira hacia arriba desde las axilas; un poste con dos montantes, al que se ata al paciente por la cintura para que haga ejercicio forzado con pesas... ${ }^{13}$.

Que fue enemigo de los cirujanos es verdad a medias, pues a quienes quiso y logró someter a la autoridad de la Facultad de Medicina en 1724, fue a los "cirujanobarberos", cobijados bajo la especialidad quirúrgica, de la cual formaban el estrato más bajo: los buenos cirujanos, que los había y muchos, quedaron también sometidos, es decir, pagaron, como ocurre a menudo, justos por pecadores. Cierto es, también, que en general les tenía algo de inquina, como lo prueba un escrito suyo de 1739 en que habla sobre la preeminencia de la medicina sobre la cirugía, redactado como una epístola: Cleon a Eudoxe: Touchant la memoire la préeminence de la médecine sur la chirurgie, que se puede encontrar en Internet por 42,45 dólares ${ }^{14}$. A ella respondió Des Rosiers con Réponse a l'écrit intitulé: Cleon a Eudoxe, touchant la preéminence prétendue des médecins sur les chirurgiens. Adressée par M. des Rosiers à M. Andry de Boisregard. Con ella vino la famosa caricatura del Homini verminoso llamando a patadas a la puerta de un cirujano-barbero para hacerle beber un purgante destinado a combatir los vermes, gracioso grabado conservado en la Biblioteca Nacional de Francia. El nombre Homini verminoso derivó del original Homo vermiculosus, con que lo bautizó Antonio Vallisnieri (1661-1730), quien era gran biólogo, discípulo de Malpighi y catedrático en Padua ${ }^{15}$.

En todo caso, una carta de Cleon a Eudoxe hubiera sido imposible pues el demagogo ateniense, sucesor de Pericles, murió junto a su vencedor, el espartano Brásidas, en la batalla de Anfipolis, que sellaría la paz entre ambas naciones, el año 422 a.C., en tanto que el astrónomo, filósofo y médico de Cnidos, (Asia Menor), famoso por su teoría de las esferas homocéntricas, en que los astros giraban alrededor de una Tierra inmóvil, nació harto después, en el 408 o en el 395 a.C.

La famosa obra de Andry llevaba el siguiente título: L'Orthopédie ou l'Art de prévenir et de corriger dans les Enfants les Difformités du Corps. Le tout par des moyens a la portée des Pères \& des Mères, \& de toutes les Personnes qui ont des Enfants a élever. Avec Figures. Y como autor: Par M. Andry, Conseiller du Roy, Lecteur \& Professeur en Médecine au College Royal, Docteurregent, \& Doyen de la Faculté de Médecine de Paris, \& $s^{16}$.

No hemos traducido el título, para que nuestros lectores se esfuercen en el francés, que es bellísima lengua, pero todos habrán podido ver que no era una obra destinada al cuerpo médico, sino "a los padres y madres, y a todas las personas que tienen niños que educar". En cuanto al término ortopedia, expresa Andry que deriva del griego orthos, "recto, según la norma", y paideia, que viene a ser "educación del paidos, o niño". La ortopedia es, así, privativa del niño, y sería absurdo hablar de "ortopedia de adultos" y un pleonasmo la "ortopedia infantil": sin embargo, ambos términos suelen usarse por quienes desconocen la lengua griega y carecen de cultura humanista, es decir, la mayoría de la llamada humanidad.

$\mathrm{Y}$ aquí llegamos al fin, pacientes lectores, al famoso 
emblema, casi universalmente aceptado, de la ortopedia, consistente en un árbol torcido, atado con una fuerte cuerda a un poste rígido, dibujo que pertenece a su colaborador e ilustrador de la obra Antoine Humblot. Al proponer el método para tratar una curvatura excesiva de la pierna de un niño, habla Andry de aplicar tan pronto como sea posible una pequeña placa de hierro en el lado cóncavo de la curvatura y fijar ésta a un vástago... para recuperar la forma de la pierna, como se usa para enderezar el tronco torcido de un árbol joven. Llamado “el árbol de Andry", se le ha querido derivar -icuándo no y cómo no!- del inefable e infaltable Hipócrates, con sus tratados sobre fracturas y articulaciones, donde usaba la tracción en la curación de las fracturas, así como el antiguo y rústico método de fijar la pierna a una férula, generalmente una tabla, para asegurar una curación sin deformaciones, lo que no siempre se lograba. Méritos de sobra tiene Hipócrates para quitarle el suyo a Andry: no llevemos más agua al molino de aquél y reconozcamos a éste los sobrados méritos que tiene para optar al título de "Padre de la Ortopedia".

\section{Otros candidatos}

En un artículo reciente Jochen Gertsner ha recordado la permanente candidatura del médico suizo Jean André Venel (1740-1791), quien en 1780 fundara la primera institución para el tratamiento de las deformidades en el cantón de Vaud, pero éste es bastante posterior a nuestro candidato $^{17}$. Sin embargo, hasta la Enciclopedia Británica avala a este sólido oponente de Andry.

Remi Kohler propone al famoso Jean Louis Petit, que muy a menudo estuvo expuesto a su (de Andry) envidioso malhumor, y que en el temprano 1705 escribió Les maladies des os (Las enfermedades de los huesos), que estima lejos más instructivo que el superficial texto de Andry.

Por su parte, el sabio Pierre Foissac, quien por ahí menciona al corresponsal Blagivi, pero negando la validez de sus teorías, ignora al mismo tiempo a Andry, pareciera proponer al famoso Portal, quien modificara la curiosa - ¿o debemos decir espantosa?- suspensión cervical ideada en 1674 por Levacher, quien también podría ser candidato, consistente en un vástago metálico que, fijado a la cintura, subía paralelo a la columna y se curvaba sobre el cráneo, al cual traccionaba hacia arriba merced a un arco abrazando la frente ${ }^{18}$ : éste y otros procedimientos invaluables son para Foissac mérito suficiente. De este Portal, cirujano y médico extraordinario, amigo de Buffon y de Franklin, cuenta su discípulo Ponseil que visitando ambos a un dispéptico renuente al régimen indicado, tomándole el maestro el pulso, lo reconvino diciéndole:

- Usted ha desayunado un huevo pasado por agua.

- ¿Qué? ¿Esto lo ha notado usted en mi pulso? -se espantó el enfermo.
- Sin duda -dijo Portal- El huevo contiene azufre, fósforo y una mezcla albuminosa que el jugo gástrico no disuelve.

De vuelta a casa, todavía estupefacto, Poinsel exclama:

- ¡Gran hombre, me postro a vuestros pies, pues habéis reconocido en el pulso que el paciente había comido huevo!

- ¡Imbécile-replicó Petit-il avait du jaune sur sa chemise! ("Imbécil, él tenía yema sobre su camisa": insisto, lectores míos, aprended francés, expresiva lengua erradicada de nuestros programas escolares por los bárbaros) ${ }^{19}$.

Esta historieta reproducida por Foissac es característica de este hombre universal, que no sólo escribía de medicina, publicando obras filosóficas y originales como "La longevidad humana y el arte de conservar la salud y prolongar la vida", "La suerte o el destino", "Higiene filosófica del alma", todas disponibles en sus ediciones originales en las librerías virtuales.

Ahora bien, volviendo a la reñida elección de Padre de la Ortopedia, sin son necesarios más votos para nuestro Homini verminoso, señalemos que la Association of Bone and Joint Surgeons, en conjunto con la Clinical Orthopaedics and Related Research, tiene instituidas tres distinciones anuales, una de las cuales lleva el nombre de Andry y tiene un premio de 25.000 dólares.

\section{Otros estudios de Andry}

Ávido de conocimientos, Andry incursionó en lo que hoy llamaríamos farmacología, publicando en 1746 una artículo sobre "el té de Europa"20, donde analizaba las propiedades de la "hierba de los leprosos", la Veronica officinalis, a la cual hasta nuestros días el ciudadano común, siempre en busca de milagros, atribuye múltiples virtudes: es "digestiva", cicatrizante, alivia la gota y el reumatismo, es expectorante y varias cosas más.

Como investigador era entusiasta admirador de Leeuwenhoek, trabajando permanentemente con el miscroscopio, y creía que gusanillos sólo así visibles -entre los que incluía, como ya vimos, los espermios- causaban varias enfermedades, entre ellas la viruela, diciendo: debemos admitir que hay animales mil veces menores que un grano de polvo, que escasamente podemos ver...sorprendentes en su extrema pequeñez, ante la cual sucumbe nuestra imaginación... ¿con qué propósito, entonces, negar su existencia, cuando la razón nos convence de su existencia de lo que no concebimos? ${ }^{21}$.

Más, siendo ante todo un humanista, se preocupó también y mucho de la lingüística y, aparte de un par de análisis sobre estudios clásicos, publicó dos tomos respecto al mal uso que sus contemporáneos hacían del francés ${ }^{22,23}$. Un par de siglos después, nosotros desesperamos del mal uso de nuestra lengua española, no sólo entre el vulgo iletrado, sino también en la prensa hablada y ihélas, mon 
Dieu!, como diría Andry, en nuestros colegas médicos.

Inquieto, virulento y genial, Andry fue un hombre cabal, que cumplió con las clásicas exigencias de tener un hijo, escribir un libro y plantar un árbol: aparte de la hija biológica, nacida de su tercer matrimonio, fue padre de otras dos, que siguen creciendo y desarrollándose hasta nuestros días; escribió al menos tres libros valiosos y plantó el árbol que lleva su nombre y que florece sin cesar.

\section{Resumen}

Homini verminoso o "Doctor Gusano" fueron los apodos ganados por Nicholas Andry, tanto por su dedicación al estudio de los gusanos intestinales como por su mal carácter, que lo llevó a atacar ferozmente a los cirujanos. Se resumen los estudios y aportes que lo llevaron a merecer el título de "Padre de la Parasitología" y a optar al de "Padre de la Ortopedia", para el cual se han propuesto también otros candidatos.

De mente inquieta, conocedor y comentador de los clásicos, incursionó en la farmacología y en la lingüística, preocupado del mal uso del francés que hacían sus contemporáneos.

Un hombre cabal, tuvo dos hijas célebres, que siguen creciendo en nuestros días; escribió al menos tres libros valiosos y plantó el "árbol de Andry", símbolo de la Ortopedia.

\section{Referencias bibliográficas}

1.- Michel L. A propos du prochain bicentenaire de l'orthopédie. Rev Chir Orthop 1938; 25: 282-6.

2.- Andrassy, le Comte Jules. Préface. En: De Bosniak Z, et Edelsheim-Gyulai. Le droit de l'enfant abandonné et le système hongrois de protection de l'enfance. 2nde édition, Imprimèrie de la Société Anonyme Athenaeum. Budapest 1912.

3.- Andry M, Conseiller du Roy, Lecteur \& Professeur en Médecine au College Royal, Docteur-regent, \& Doyen de la Faculté de Médecine de Paris, \&s. De la generation des vers dans le corps de l'homme, de la nature et des espéces de cette maladie, de moyens de l'en préserver et de le guérir. Avec trois lettres écrites a l'auteur sur le sujet des vers, les deux premières d'Amsterdam, par M. Nicolas Hartsoeker, et l'autre de Rome, par M. Georges Blagiv. Chez La Veuve Alix, au-dessus de la rue des Noyers, au Grisson. Lambert \& Durand, à la Sagesse, \& 1 Saint Landry, Paris 1700

4.- Doetsch R N. Benjamin Marten and his "New theory of consumptions". Microbiol Rev 1978; 42 (3): 521-8.

5.- Andry M, Conseiller du Roy, Lecteur \& Professeur en Médecine au College Royal, Docteur-regent, \& Doyen de la Faculté de Médecine de Paris, \&s. Vers solitaires et autres de diverses espèces, dont il est traité dans le livre De la Génération des vers, représentés en plusieurs planches, ensemble plusieurs remarques importantes sur ce sujet.
Laurent d'Houry, éditeur. París 1718.

6.- Baglivi G. Complete Dictionary of Scientific Biography. 2008. Encyclopedia.com. 9 Sep. 2011 http://www. encyclopedia.com

7.- Véritail M. Ma vie sur les bateaux. J.L. Beauchamp, Éditeur. Marseille 1874.

8.- Villanúa L. Historia regocijante de el Gran Don Medín Medina. Imprenta de Galo Saéz, Mesón de Paños 8, Madrid 1930; pp. 48; 173-4.

9.- Kohler R. Nicolas Andry de Bois-Regard (Lyon 1658-Paris 1742): The inventor of the word "orthopaedics" and the father of parasitology. J Child Orthop 2010; 4 (4): 349-55.

10.- Havens G R. Voltaire, Francois-Marie Arouet de. Collier's Encyclopedia. Crowell Clloier and MacManus, Inc, Phi 1967; 23: 195-9.

11.- Voltaire. Novelas escogidas. Traducción del francés por el abate José Marchena. Editorial Iberia, S.A. Barcelona 1967.

12.- Penny cyclopaedia of the Society for the Diffusion of Useful Knowledge, Great Britain; 5: 182.

13.- Kirmissen M. Précis de chirurgie infantile. Masson \& Cie., Éditeurs, Paris 1906; pp.536-52.

14.- Andry M. Cleon a Eudoxe: Touchant la memoire la préeminecne de la médecine sur la chirurgie. Paris 1739. Facsímil de Junio 13, 2009, Amazon.com, con la advertencia: This book is a facsimile reprint and may contain imperfections such as marks, notations, marginalia and flawed pages.

15.- Generali D, Vallisneri A. Gli anni della formazione e le prime ricerche. Firenze, Olschki, 2007.

16.- Andry M, Conseiller du Roy, Lecteur \& Professeur en Médecine au College Royal, Docteur-regent, \& Doyen de la Faculté de Médecine de Paris, \&s. L'Orthopédie ou l'Art de prévenir et de corriger dans les Enfants les Difformités du Corps. Le tout par des moyens a la portée des Pères \& des Mères, \& de toutes les Personnes qui ont des Enfants a élever. Avec Figures. Chez La Veuve Alix, au-dessus de la rue des Noyers, au Grisson. Lambert \& Durand, à la Sagesse, \& 1 Saint Landry. Paris, Rue Saint Jacques, 1741

17.- Gertsner Burns J. Biografía de Nicolás Andry. Rev Col Or Tra 2009; 23 (2): 82-3.

18.- Jackson G E. Ortopedia appliances. Collier's Encyclopedia. Crowell Collier and MacMillan, Inc, Phi 19677; 18: 232-5.

19.- Foissac, Le docteur P. Hygiène des saisons. J-B Balliere et fils, Rue Hautefeuille, 19, près le Boulevard Saint Germain, p.41. Paris 1884 .

20.- Andry N. Le Thé de l'Europe, ou les Propriétés de la véronique, tirées des observations des meilleurs auteurs et surtout de celles de M. Francus, médecin allemand. Laurent d'Houry, éditeur. Paris 1746

21.- Correia C P. The ovary of Eve. Egg and sperm and preformation. Chicago 1997, University of Chicago Press; pp. 74-6.

22.- Andry N. Réflexions sur l'usage présent de la langue française ou Remarques nouvelles et critiques touchant la politesse du langage. París 1689.

23.- Andry N. Suite des réflexions critiques sur l'usage présent de la langue françoise. París 1693. 